\title{
Theta Responses Are Involved in Lexical-Semantic Retrieval during Language Processing
}

\author{
Marcel C.M. Bastiaansen ${ }^{1,2}$, Marieke van der Linden ${ }^{1}$, \\ Mariken ter Keurs ${ }^{2}$, Ton Dijkstra ${ }^{3}$, and Peter Hagoort ${ }^{1}$
}

\begin{abstract}
Oscillatory neuronal dynamics, observed in the human electroencephalogram (EEG) during language processing, have been related to the dynamic formation of functionally coherent networks that serve the role of integrating the different sources of information needed for understanding the linguistic input. To further explore the functional role of oscillatory synchrony during language processing, we quantified event-related EEG power changes induced by the presentation of open-class (OC) words and closed-class (CC) words in a wide range of frequencies (from 1 to $30 \mathrm{~Hz}$ ), while subjects read a short story.
\end{abstract}

\section{INTRODUCTION}

A central characteristic of language comprehension is that very rapidly very different sources of information, including information about form, syntax, and meaning of words and sentences, have to be accessed and combined. Brain imaging studies have shown that a large number of brain areas are involved in processing each of these relevant types of information (e.g., Kaan \& Swaab, 2002; Indefrey \& Levelt, 1999; Price, Indefrey, \& Van Turennout, 1999). Because at a given moment the different bits of information that are processed in different parts of the brain must be integrated to obtain a unified concept of the language input, the different brain areas involved have to communicate with each other. A fundamental issue in research on the neurocognition of language comprehension is therefore how the dynamic binding of the distributed nodes of the language network takes place, such that an adequate mapping of form onto meaning results.

A good candidate mechanism for such dynamic network formation is that of synchronization and desynchronization of oscillatory neuronal activity. The notion that synchronous, oscillatory neuronal activity plays an important role in dynamically linking brain areas has gained firm experimental footing after the initial experi-

\footnotetext{
${ }^{1}$ F. C. Donders Centre for Cognitive Neuroimaging, Nijmegen, The Netherlands, ${ }^{2}$ Max Planck Institute for Psycholinguistics, ${ }^{3} \mathrm{Nijmegen}$ Institute for Cognition and Information
}

Word presentation induced three oscillatory components: a theta power increase $(4-7 \mathrm{~Hz})$, an alpha power decrease (10$12 \mathrm{~Hz})$, and a beta power decrease (16-21 Hz). Whereas the alpha and beta responses showed mainly quantitative differences between the two word classes, the theta responses showed qualitative differences between OC words and CC words: A theta power increase was found over left temporal areas for OC words, but not for CC words. The left temporal theta increase may index the activation of a network involved in retrieving the lexical-semantic properties of the OC items.

ments described by Gray, König, Engel, and Singer (1988) and Singer (1993). Recent experimental findings strongly suggest that synchronous oscillations also play an important role during the execution of tasks engaging a variety of cognitive functions. Although much remains to be done, these and other studies on event-related changes in oscillatory electroencephalogram (EEG)/ magnetoencephalogram (MEG) activity start to reveal the contours of a functional characterization of the oscillatory activity in different frequency bands (although there is obviously no one-to-one correspondence between frequencies and neurocognitive functions). The general pattern of findings roughly seems to indicate that depending on its scalp topography, the modulation of activity in the $10-\mathrm{Hz}$ frequency range (i.e., alpha and $\mathrm{mu}$ ) is related to the processing of either sensory input or motor output (e.g., Bastiaansen \& Brunia, 2001; Fries, Reynolds, Rorie, \& Desimore, 2001; Klimesch, 1999; Foxe, Simpson, \& Ahlfors, 1998). Activity in the beta frequency range (roughly $15-25 \mathrm{~Hz}$ ) has been linked to active inhibition of ongoing processes, as indexed, for instance, in the postmovement beta rebound (Pfurtscheller \& Lopes da Silva, 1999; Salmelin \& Hari, 1994; Pfurtscheller, 1992). For both alpha and beta band activity, it holds that the amplitude of these oscillations is inversely related to active processing, such that amplitude reductions stand for active processing, whereas increases stand for cortical idling and/or inhibition. The opposite holds for the two other frequency bands that have been extensively studied, that is, the theta and 
gamma bands. Here it generally holds that amplitude increases are related to the active processing of information. Gamma oscillations have been linked to topdown perceptual processes (e.g., Rodriguez et al., 1999; Tallon-Baudry \& Bertrand, 1999). Its role in the integration (binding) of information within relatively small patches of cortex has been extensively studied in the visual system (Fries et al., 2001; see also the reviews in Roskies, 1999). Theta oscillations (and to some extent also gamma) have been mostly implicated with storage and retrieval of information from long-term memory (e.g., Burgess \& Ali, 2002; Fell et al., 2001; Klimesch et al., 2001; Klimesch, 1999). In addition, working memory processes have been related to theta (e.g., Bastiaansen, Posthuma, Groot, \& de Geus, 2002; Jensen \& Tesche, 2002; Tesche \& Karhu, 2000; Kahana, Sekuler, Caplan, Kirschen, \& Madsen, 1999).

Given these findings, and given the importance of a rapid integration of different sources of information for language processing, it seems reasonable to hypothesize that synchronous oscillations may play a crucial role during language processing as well. However, until recently only very few studies have experimentally addressed the functional role of synchronous oscillatory activity during language processing (Weiss, Müller, \& Rappelsberger, 2000; Pulvermüller, 1999; Pulvermüller, Eulitz, et al., 1996; Pulvermüller, Preissl, Lutzenberger, \& Birbaumer, 1996). This may partly be explained by the fact that appropriate research methodologies have only recently become widely available. As we pointed out in detail elsewhere (Bastiaansen \& Hagoort, 2003), standard event-related potential (ERP) analysis yields only a limited view on oscillatory phenomena because the latter are for a large part not phase-locked to the event in question, and thus largely cancel out when single trials are averaged. To properly capture synchronization and desynchronization of oscillatory neuronal activity, analyses must focus on the event-related changes in amplitude and phase of frequency-specific EEG/MEG rhythms at the single-trial level. The amplitude of rhythmic activity is of interest because the synchronization of local groups of neurons, the activities of which are picked up by one and the same sensor, will result in larger EEG/MEG amplitudes for that sensor (e.g., Nunez, 1995). It follows that local changes in synchrony of oscillatory firing patterns lead to changes in amplitude of rhythmic EEG/MEG activity. The phase of EEG/MEG oscillations is important because synchronization of oscillatory firing patterns between spatially distant neuronal populations lead to increases in (phase) coherence between two (or more) concurrently measured EEG or MEG signals (e.g., Lachaux, Rodriguez, Martinerie, \& Varela, 1999; for a comprehensive review of available methodologies for analyzing oscillatory synchrony, see Varela, Lachaux, Rodriguez, \& Martinerie, 2001).

In this article we aim to further characterize the functional role of oscillatory synchrony in language comprehension. In a previous study (Bastiaansen, van Berkum, \& Hagoort, 2002a), we quantified amplitude changes in the alpha and theta rhythms of the EEG, recorded while participants read correct Dutch sentences that were presented word by word in the visual modality. We found widely distributed theta increases following individual words, and a gradual theta increase over the sentence. The former may reflect the formation of a network involved in lexical processing, whereas the latter may be related to increased working memory load as the individual words in a sentence incrementally converge into an overall understanding of the meaning of the sentence. In a subsequent study (Bastiaansen, van Berkum, \& Hagoort, 2002b), we investigated the effects of syntactic violations on theta oscillations. Comparing sentences containing either a grammatical gender violation or a number violation to correct sentences, we found that syntactic violations elicited larger theta responses at frontal electrodes, with a left-hemisphere dominance following number violations and a righthemisphere preponderance following gender violations. Adjacent frequency bands (i.e., delta and alpha) did not show such reactivity, indicating that also in this study the effects were restricted to the oscillatory activity in the theta band. Similar results were found in a third study, where theta increased over the frontocentral midline following semantic violations in sentences (Hagoort, Hald, Bastiaansen, \& Petersson, 2004). Together, these findings raise the question of why theta power consistently reacts to different aspects of language processing. This question may be partly answered by realizing that there is a large overlap between the language system on the one hand, and the declarative and working memory systems on the other hand. Given that a robust relation has been found between theta power increases and memory encoding/retrieval (e.g., Burgess \& Ali, 2002; Fell et al., 2001; Klimesch et al., 2001; Klimesch, 1999) and working memory (e.g., Bastiaansen, Posthuma, et al., 2002; Jensen \& Tesche, 2002; Tesche \& Karhu, 2000; Kahana et al., 1999), the findings of theta power correlating various linguistic manipulations may index processes at the interface between memory and language, such as retrieval of language-related visual information (e.g., stored word forms), or retrieval of lexico-semantic information.

In the present article we exploit the functional differences between the primarily semantic function of words belonging to the open class (OC), such as nouns, verbs, and adjectives, and the primarily syntactic nature of words belonging to the closed class (CC), such as determiners, conjunctions, and prepositions. If our hypotheses concerning the role of oscillatory synchrony in language comprehension are correct, processing OC words should lead, among others, to the emergence of a network involved in assigning meaning to the lexical items. Because this mainly involves retrieving information stored in long-term memory, such a network would 
likely operate by synchronizing the activity of its constituent neurons at theta frequencies. Based on our current understanding of oscillatory phenomena, we would therefore expect increases in theta-band activity to accompany the processing of OC words. For the semantically impoverished CC words, this should be much less obvious. On the other hand, the more pronounced syntactic function of CC words may lead to increased activation of a network involved in syntactic (lemma level) analysis. For the other frequency bands that we consider in this article (i.e., alpha and beta), we expect power decreases to accompany the processing of OC and CC items alike, because oscillatory activity in these frequency ranges is generally inversely related to (attentional demands involved in) the processing of sensory information (see above), which are similar for both types of items in the present experiment.

At the same time, the current approach of quantifying oscillatory synchrony may yield new information with respect to the claim that $\mathrm{OC}$ words and $\mathrm{CC}$ words are represented and/or accessed differently. This claim was initially put forward on the basis of (1) neuropsychological data showing differential impairments for the two word classes for specific patient groups (e.g., Friederici, 1983, 1985; Benson, 1979), and (2) behavioral data showing a right visual field advantage for CC compared to OC words after tachistoscopic half-field presentation (e.g., Mohr, Pulvermüller, \& Zaidel, 1994; Chiarello \& Nuding, 1987; Bradley \& Garrett, 1983). Although these studies do suggest separate lexical representations and/ or lexical access routes for the two word classes, the claim would be much stronger if one could demonstrate differences in neuronal implementation between OC and CC words. This issue has been addressed by a substantial number of ERP studies. Because good reviews are available (e.g., Münte et al., 2001; Brown, Hagoort, \& ter Keurs, 1999), we only briefly summarize the main results of these studies here, ignoring those ERP components that were reported in only one or a few articles. Early studies (e.g., Neville, Mills, \& Lawson, 1992; van Petten \& Kutas, 1991) suggested that OC and CC words elicit qualitatively different ERP components: an N280 for CC words, an N400 for OC words, and a late component, termed N400-700 for CC words. Subsequent experiments showed that the N280 was also evoked by OC words, with the latency of the negative peak being dependent on word length and word frequency (King \& Kutas, 1995; Nobre \& McCarthy, 1994; Neville et al., 1992). Therefore, King and Kutas (1995) termed this component lexical processing negativity (LPN). However, other studies (ter Keurs, Brown, \& Hagoort, 2002; Brown et al., 1999; ter Keurs, Brown, Hagoort, \& Stegeman, 1999) also found an N280 to OC words, but did not find this component to be sensitive to word frequency and word length. Instead, these studies reported length- and frequency-independent amplitude and latency differences between the two word classes, which was taken to be the earliest electrophysiological reflection of the availability of word class information.

The next relevant ERP component, the N400, was shown to be elicited not only by OC, but also by CC words, albeit of a smaller amplitude for CC words (e.g., Münte et al., 2001; King \& Kutas, 1995; Nobre \& McCarthy, 1994; van Petten \& Kutas, 1991). This leaves the N400-700 as the only ERP component that yields a qualitative distinction between the two word classes, because it has been found only for CC words, both in sentence context (Brown et al., 1999; Osterhout, 1997; King \& Kutas, 1995; Neville et al., 1992; van Petten \& Kutas, 1991) and in word lists (ter Keurs et al., 2002; Münte et al., 2001). The N400-700 has been interpreted as reflecting syntactic processing, more specifically as indicating an expectation for the occurrence of an OC word (ter Keurs et al., 2002; Münte et al., 2001). Its very long latency dictates that it must be a postlexical process.

In sum, the ERP literature suggests that the functional distinction between $\mathrm{OC}$ words and $\mathrm{CC}$ words is mirrored by a neurophysiological distinction. However, the exact nature of this distinction needs further clarification. Our current approach, based on the quantification of patterns of oscillatory synchrony, may shed new light on this issue. We therefore subjected EEG data of a previously published ERP study (Brown et al., 1999) to a new analysis. In this experiment, participants passively read a short story, presented word by word on a monitor. EEG responses were extracted separately for words belonging to the OC and words belonging to the CC. A waveletbased time-frequency analysis (Tallon-Baudry, Bertrand, Delpuech, \& Pernier, 1996) was used to quantify eventrelated amplitude changes of EEG oscillations. Note that, as we pointed out above, amplitude changes index changes in oscillatory synchrony in local cortical networks. Therefore, what we can obtain from the present analysis is information about synchronization changes within individual "nodes" of the language processing network. Different tools, such as coherence analysis, would be needed to obtain information about synchronization between such network elements. In addition to a participant analysis, in which we compared the patterns of synchrony induced by OC words and CC words over individuals, we performed an item analysis to verify whether class differences in oscillatory synchrony are attributable to lexico-statistical factors such as word length and word frequency.

\section{RESULTS \\ Participant Analysis}

For each word class, time-frequency representations (TFRs) were averaged over trials for each subject. Through visual inspection of the data (see Figure 1), three time/frequency components were identified that 
Figure 1. TFRs and scalp topographies of the power changes elicited by OC words (left) and CC words (right). TFRs are given for a representative channel (Fz), with blue representing power decreases and red depicting power increases. Black rectangles in the TFRs indicate the time-frequency intervals selected for statistical analysis. Scalp topographies (same color coding) are given for these time-frequency intervals, for OC and CC words separately. Green dots indicate electrode positions. Spacing of isopercentage lines is $2 \%$ power change. Note the qualitative difference in scalp topography for the theta power changes.

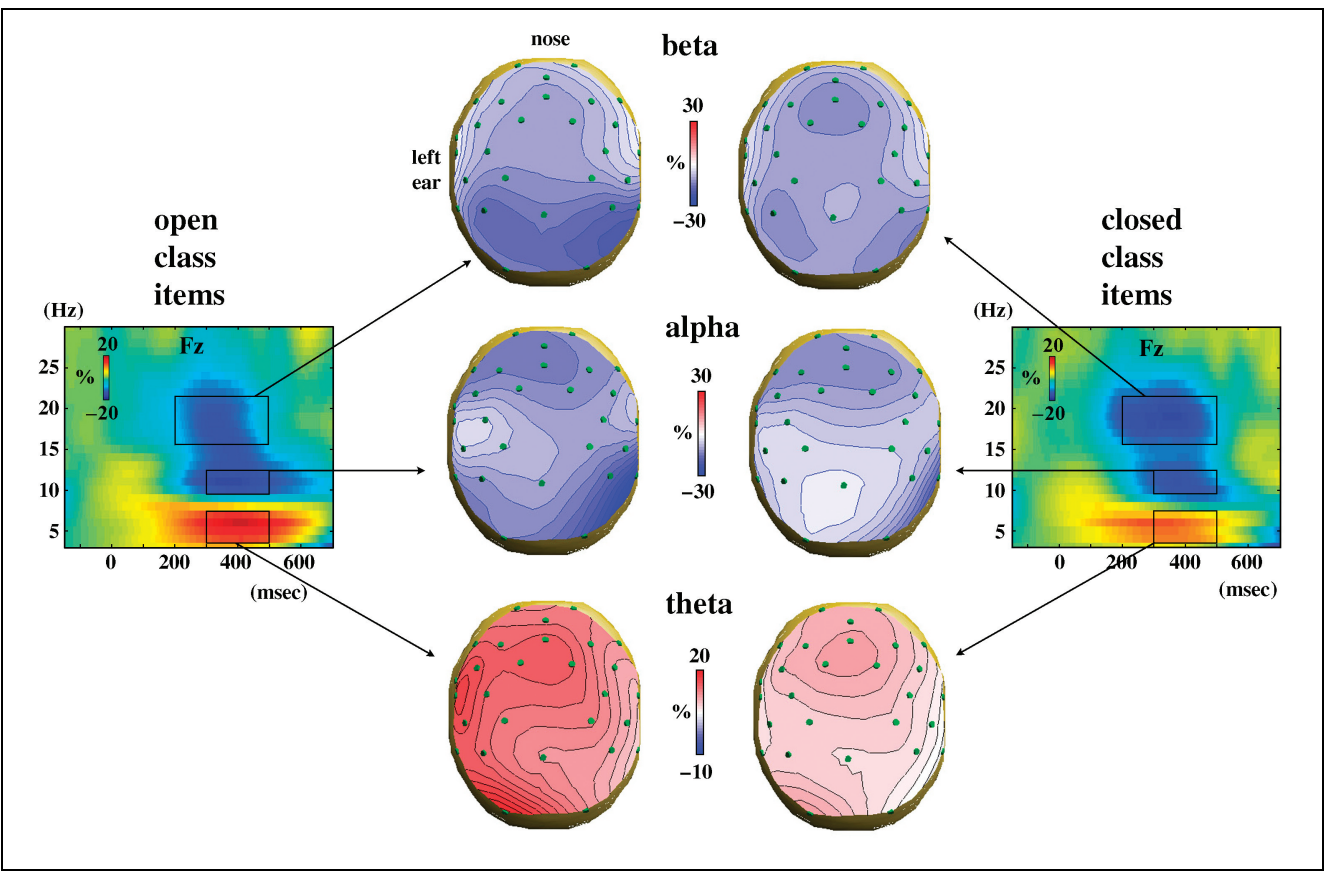

showed a clear reactivity for both word types: theta (4$7 \mathrm{~Hz}$ ), with a peak power increase between 300 and 500 msec poststimulus; alpha $(8-10 \mathrm{~Hz})$, with a decrease peaking between 300 and $500 \mathrm{msec}$ poststimulus, and beta $(16-21 \mathrm{~Hz})$, with a broad peak between 200 and $500 \mathrm{msec}$ poststimulus. The resulting time/frequency "rectangles" are depicted in Figure 1.

Within each of these time/frequency ranges, power values were averaged at the individual subject level, and then averaged over participants. From the resulting scalp topographies (cf. Figure 1), we identified by means of visual inspection, for each time-frequency range, a number of regions of interest (ROIs) corresponding to the topographic minima or maxima. Data at electrodes belonging to a particular ROI were averaged at the individual subject level and used as dependent variables in the statistical analyses. ROIs were as follows: for the theta range: midfrontal (Fz, FC1, FC2), left temporal (T3, TFC1, TPC1), and left occipital (being O1); for the alpha range: frontal midline $\left(\mathrm{Fz}^{\prime}\right.$ and $\left.\mathrm{Fz}\right)$, and right occipitotemporal (P4, T6, and $\mathrm{O} 2$ ); for the beta band midfrontal (Fz, FC1, FC2), left occipitotemporal (P3, T5, O1), and right occipitotemporal (P4, T6, and $\mathrm{O} 2)$.

An ANOVA for repeated measures was performed for each time/frequency range, with the factors word class and ROI. Significant interactions were clarified by breaking them down into simple effects. Where necessary, degrees of freedom were corrected using the Greenhouse Geisser epsilon.

Whereas the scalp topographies of the power changes for the two word classes look qualitatively roughly similar in the alpha and beta bands (although appearing to be larger for OC words), visual inspection suggests that in the theta band the scalp topography for OC words is qualitatively different from the topography for $\mathrm{CC}$ words in that an additional component seems to be present at left temporal electrode positions.

\section{Beta Power Decreases}

Statistical analysis of the beta power decrease shows an overall power decrease after word presentation, $F(1,12)=54.95, p<.001$. There were no significant main effects of word class and ROI. However, a Word Class $\times$ ROI interaction, $F(2,24)=6.06, p=.017$, indicates that the beta power decrease is larger following the presentation of OC words compared to CC words both at the left occipitotemporal, $F(1,12)=11.45, p=$ .005 , and the right occipitotemporal, $F(1,12)=9.31$, $p=.010$, ROIs, not at the midfrontal ROI, $F(1,12)=$ $0.628, p=.443$.

\section{Alpha Power Decreases}

As for the beta component, statistical analysis of the alpha power decrease shows an overall power decrease after word presentation, $F(1,12)=20.67, p=.001$. There were no significant main effects of word class and ROI. A Word Class $\times$ ROI interaction, $F(1,12)=$ $9.93, p=.008$, indicates that the alpha power decrease is larger following the presentation of OC words compared to CC words at the right occipitotemporal ROI, $F(1,12)=9.89, p=.008$, but not at the frontal midline ROI, $F(1,12)=0.875, p=.368$. 


\section{Theta Power Increases}

The ANOVA on the theta power increase indicates that overall, word presentation elicits a theta power increase, $F(1,12)=22.10, p=.001$, which is larger for OC words than for CC words: main effect of word class, $F(1,12)=$ $34.05, p<.001$. Furthermore, an interaction of Word Class $\times$ ROI, $F(2,24)=4.25, p=.042$, indicates that this effect is restricted to left temporal, $F(1,12)=38.79, p<$ .001 , and left occipital, $F(1,12)=16.58, p=.002$ ROIs, word class differences being nonsignificant at the midfrontal ROI, $F(1,12)=3.43, p=.098$.

Because the theta increase at the left temporal ROI is substantial for OC words but almost nonexistent for CC words (19.9\% vs. $3.4 \%$ power increase, respectively; see also Figure 1), we used single-sample $t$ tests to verify whether the power increase in this ROI differs from zero. This was found to be the case for the OC words $(p<.001)$. The theta power increase in the left temporal ROI was not significantly different from zero for CC items $(p=.179)$.

\section{Item Analysis}

The participant analysis showed a qualitative difference between the two word classes in the scalp topography of the theta power increases: At left temporal electrode positions, a theta increase was present for OC words but not for CC words. Because in behavioral data and ERP data qualitative differences between the two word classes can often be attributed to differences in lexicostatistical properties, such as word length and word frequency, we performed a regression analysis at the single-item level to establish whether these word properties can also account for the present theta effects.

TFRs for each individual item were averaged over participants, separately for the two word classes. From these item averages, we then extracted the average power change in a time window from 300 to $500 \mathrm{msec}$ after word onset, and a frequency window from 4 to $7 \mathrm{~Hz}$ for each EEG channel. To determine the relation between the amount of power change an item elicited on the one hand, and its length and frequency on the other hand, the values thus obtained were entered as dependent measure in a multiple regression analysis with length and scarcity ( $5-\log$ frequency) as quantitative predictors and word class (open vs.closed) as a categorical predictor. This analysis was performed four times: once on an average of all electrodes, and once for each of the three ROIs defined for the theta band.

Figure 2 presents scatterplots and regression lines of the average percent power changes in the theta range for individual words, as a function of word length and word frequency. Visual inspection of Figure 2 suggests that word length and word frequency do not have a substantial influence on the amount of theta power change.

Table 1 presents the parameters of the multiple regression equations obtained from the four regression analyses (three ROIs, and the average over all electrodes), as well as the percentage variance explained by the respective regression models. Note that these percentages are very low, in all cases well below 1\%, indicating that word length and word scarcity have very little influence on theta power increases.

On the regression parameters two types of statistical tests were performed. First, to assess whether word

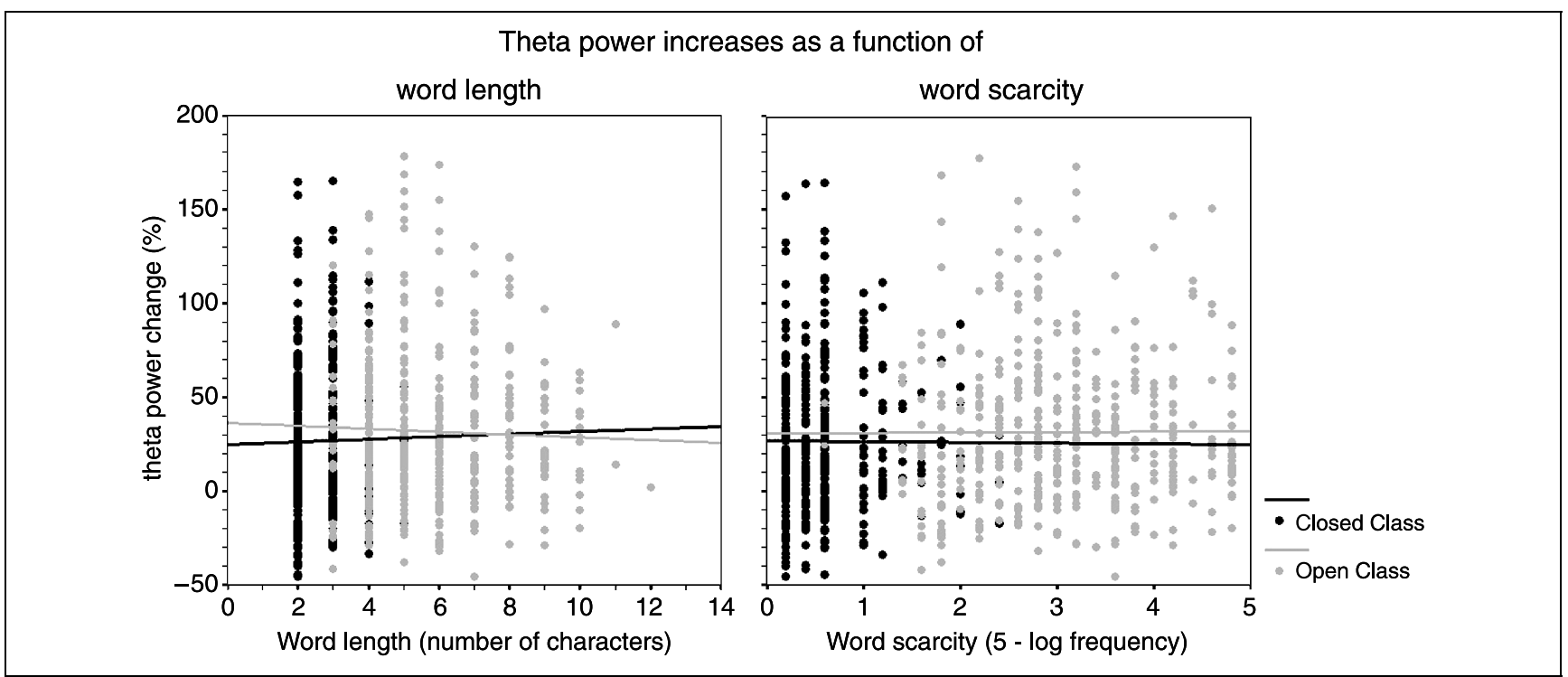

Figure 2. Scatterplots and regression lines showing the effects of word length and word frequency on the theta power increases, given separately for OC (gray circles) and CC (black circles) words. The $y$-axis represents theta power increase computed for individual trials, averaged over subjects and electrodes. 
Table 1. Regression Parameter of the Items Analysis on Theta Power Increases

\begin{tabular}{|c|c|c|c|c|c|c|c|}
\hline \multirow[b]{3}{*}{ Analysis } & \multicolumn{6}{|c|}{ Parameter } & \multirow{3}{*}{$\begin{array}{c}R^{2}(\% \text { Variance Explained } \\
\text { by Regression Model) }\end{array}$} \\
\hline & \multicolumn{2}{|c|}{ Intercept } & \multicolumn{2}{|c|}{ Coefficient Word Scarcity } & \multicolumn{2}{|c|}{ Coefficient Word Length } & \\
\hline & $O C$ & $C C$ & $O C$ & $O C$ & $C C$ & $C C$ & \\
\hline All electrodes & $27.8^{*}$ & $18.2 *$ & 2.86 & -1.39 & 3.16 & -2.87 & 0.4 \\
\hline Midfrontal ROI & $26.1^{*}$ & $27.9^{*}$ & 2.88 & -0.88 & -0.63 & -0.20 & 0.2 \\
\hline Left temporal ROI & $34.4^{*}$ & 7.9 & 1.12 & -1.02 & 2.18 & -3.2 & 0.5 \\
\hline Left occipital ROI & $30.4^{*}$ & $18.0^{*}$ & 2.94 & $1.99 * *$ & 2.4 & -3.12 & 0.6 \\
\hline
\end{tabular}

${ }^{*} p<.01$, different from zero.

$* * p=.084$, marginally significant.

length and word frequency differentially affect theta reactivity to OC and CC words, we tested whether the coefficients of word length and word scarcity of the regression equations for $\mathrm{OC}$ words and $\mathrm{CC}$ words differed from each other by means of an omnibus generalized linear model (GLM) test for the homogeneity of coefficients. For each of the four regressions analyses, the results indicate that none of the coefficients of the regression equations differed between the two word classes (all $p$ values $>.6$ ).

Second, to establish whether word length and word frequency have any influence at all on the power changes induced by OC and CC words, we tested whether the coefficients of the regression lines differed from zero. The results from this analysis are summarized in Table 1, and indicate that none of the regression slopes differed from zero, with one exception: For OC words the (positive) coefficient for word length was marginally significant at the left occipital ROI, indicating slightly larger power values in this ROI with longer words.

Note that the intercepts did differ from zero in all the regression equations, except in the equation for CC items in the left temporal ROI. This confirms the pattern of statistical significance of the theta power increases observed in the participant analysis.

\section{DISCUSSION}

To demonstrate a functional role of synchronous oscillatory neural activity in dynamically forming integrative networks involved in the processing of primarily semantic OC words and primarily syntactic CC words, we quantified the patterns of oscillatory EEG synchrony induced by words of both classes.

Our analyses showed the existence of oscillatory responses in the EEG, in three distinct frequency bands: theta $(4-7 \mathrm{~Hz})$, alpha $(10-12 \mathrm{~Hz})$, and beta $(16-21 \mathrm{~Hz})$. Note that because of the filter settings used in the EEG recordings, we were not able to analyze activity in the gamma frequency range, although there are experimen- tal data (Hagoort et al., 2004; Pulvermüller, Eulitz, et al., 1996) that suggest a role of gamma band activity in language processing. In the beta band, a power (i.e., squared amplitude) decrease was observed, with maxima peaking around 200-500 msec after word onset over midfrontal and over bilateral occipitotemporal areas for both word classes. Over the latter areas, the beta power decrease was stronger following $\mathrm{OC}$ words compared to CC words. In the alpha band, a similar power decrease was observed, albeit with slightly different time course and scalp topography: Here the power decrease started later, peaking around 300-500 msec after word onset, and was restricted to the midfrontal and right occipitotemporal areas. Finally, in the theta frequency range we found a power increase, peaking between 300 and $500 \mathrm{msec}$ poststimulus, and which consisted of three topographic components: (1) a left occipital component that was stronger for OC words than for CC words; (2) a midfrontal component that was identical in magnitude for the two word classes; and (3) a left temporal component that was elicited only by the processing of OC words. A regression analysis at the level of individual items indicated that the theta power increases did not vary with word length and word frequency, with one exception: At the left occipital electrode, power increases following OC words showed a (marginally significant) positive correlation with word length. Overall, the present results are consistent with those from our previous studies (Bastiaansen, van Berkum, et al., 2002a, $2002 \mathrm{~b}$ ), in which we also found theta power increases together with alpha power decreases during language processing. The concurrent beta power increase is a new element because we did not previously investigate this frequency band.

In line with our previous interpretation (Bastiaansen, van Berkum, et al., 2002a, 2002b), it seems likely that the alpha power decrease is related to the sensory processing of the incoming visual information. This fits the general observation that alpha-band activity is related to the thalamocortical transmission of sensory information (e.g., Bastiaansen \& Brunia, 2001; Foxe et al., 1998; 
Lopes da Silva, 1991; Steriade, Gloor, Llinás, Lopes da Silva, \& Mesulam, 1990).

The beta power decrease is more difficult to interpret because little is known about the neural basis and functional significance of the reactivity of beta-band rhythms in paradigms that do not involve motor tasks. Given the similarity in scalp topography and reactivity to word class of this effect compared to the alpha-band effect, one could suggest that the beta response is also related to the sensory processing of the visual input. However, Weiss and Rappelsberger (1996) found differences in beta-band coherence between concrete and abstract nouns, suggesting a functional rather than a mere sensory role of beta-band activity in language processing. Of interest here is that the beta reactivity found by Weiss and Rappelsberger was in a frequency band well below the beta reactivity found in the present experiment (13-18 Hz vs. $16-21 \mathrm{~Hz}$, respectively). This may indicate that these two beta phenomena are qualitatively different in that they do not necessarily share neurophysiological sources nor have similar functionality. In sum, the lack of sufficient experimental data on beta reactivity during language processing makes it difficult to interpret the present findings in this frequency band.

The most interesting findings of the present study are in the theta frequency range, because only there do we find qualitative differences in reactivity between the two word classes, in addition to quantitative differences.

As we argued in the Introduction, power changes, as opposed to changes in coherence, are most likely caused by local changes in neural synchrony. The three topographically distinguishable theta components found in the present experiment are therefore considered to reflect increased activity within individual nodes of the language processing networks, and, consequently, they may have different functional correlates. It should be noted that witnessing the inconsistencies in the findings of ERP studies addressing the distinction between OC words and CC words, the effects reported here need to be replicated before any strong claims can be made. Nevertheless, below we will attempt to delineate the functional correlates of each of the theta components we found.

The left occipital component may be related to the processing of the visual word form (e.g., Cohen et al., 2000). This interpretation is supported by two aspects of the results. First, this component is present for both word classes, as one would expect because the word form of every word, OC or CC alike, has to be represented. Second, the theta power increase at left occipital areas is of a larger magnitude following OC words (which are longer on the average). Furthermore, within the OC there is a similar tendency (albeit small, and only marginally significant) of longer words to elicit larger theta power increases in this region. These findings are in line with the idea that longer words require the synchronization of a larger number of network elements (neurons). A larger number of synchronized neurons leads to the observed differences in theta power increase. Experiments specifically designed to test the possibility of a relation between occipital theta increases and word length would be helpful in determining whether this interpretation is viable.

The frontocentral midline component is of similar magnitude in both word classes. Because frontal (midline) theta is often implicated in working memory (e.g., Kahana, Seelig, \& Madsen, 2001; Tesche \& Karhu, 2000; Gevins, Smith, McEvoy, \& Yu, 1997) it is tempting to conclude that this component is a reflection of (verbal) working memory processes involved in forming a memory trace of the sentence. An alternative interpretation would be that this component is related to the processing of syntactic (lemma level) information. This would be roughly in line with previous findings showing frontal theta increases following syntactic violations (Bastiaansen, van Berkum, et al., 2002b), although in that study we investigated syntactic parsing operations at the sentence level. Experiments in which syntactic processing load (both at the lemma level and at the sentence level) and working memory load are manipulated independently would be necessary to further delineate the functional correlates of the frontal theta effect.

Finally, the left temporal theta component represents a qualitative difference between OC words and CC words. Because it is found only following OC words, it might be related to retrieving the meaning of the lexical item in question. Support for this hypothesis stems from numerous studies demonstrating increases in theta power during retrieval of information from long-term memory (e.g., Burgess \& Ali, 2002; Klimesch et al., 2001; Burgess \& Gruzelier, 2000; Klimesch, 1999). Moreover, this interpretation fits the results of brain imaging studies of language comprehension that show activations of the left middle/superior temporal areas during the retrieval of the semantic code (e.g., Scott, Blank, Rosen, \& Wise, 2000; Hagoort et al., 1999; Binder, Frost, Hammeke, Rao, \& Cox, 1996). Similarly, a meta-analysis of 58 brain imaging (mostly positron emission tomography) studies into language production (Indefrey \& Levelt, 1999) shows that lexical selection on the basis of a concept (i.e., the opposite process) is also associated with left middle temporal gyrus activation. It is tempting to relate the activation of the left middle and superior temporal areas found with neuroimaging techniques to the left temporal theta responses found in the present data. However, the relation between hemodynamic responses and oscillatory EEG phenomena is only poorly understood at present.

Apart from the exact functional nature of each of the three topographically distinguishable theta components, the very fact that the major differences between OC and CC words were found in the theta frequency range is interesting in itself. It is consistent with the results from 
previous studies (Hagoort et al., 2004; Bastiaansen, van Berkum, et al., 2002a, 2002b), in which theta power was found to react on a variety of linguistic manipulations. As we noted in the Introduction, this may at least partially be explained by the large overlap in the neural systems underlying mnemonic processes (which robustly elicit theta power increases) and linguistic processes. Therefore, the theta power changes reported here may represent aspects of language processing viewed through a "mnemonic filter." It has been argued repeatedly that memory-related, scalp-recorded theta oscillations may be indicative of feedback loops between the neocortex and medial temporal lobe (MTL) memory system (for reviews, see Bastiaansen \& Hagoort, 2003a; Kirk \& Mackay, 2003; Miller, 1991). Reasoning along these lines, the present finding of larger theta power following OC words compared to CC words over temporal recording sites therefore suggests an increase in activity in MTL temporal cortex loops. This is further supported by the non-phase-locked (i.e., induced) nature of the theta effects, which is consistent with the idea that it indexes a modulation of ongoing activity. Although scalp EEG data cannot be conclusive on this hypothesis (because at the scalp one can only measure activity at the "cortical end" of a cortico-hippocampal loop), supportive evidence for this hypothesis stems from intracranial recordings from within the MTL, which have shown larger field potentials for open-class words compared to closed-class words (Nobre \& McCarthy, 1995).

With respect to the latency of the theta effects, a word of caution is warranted. The temporal resolution of timefrequency analysis is inherently relatively poor compared to ERP measures (e.g., Knosche \& Bastiaansen, 2002), with lower frequencies having poorer temporal resolution (in the order of a few hundred milliseconds) than higher frequencies. In addition, the use of sliding windowing techniques as in the present wavelet analysis leads to some additional temporal smearing of effects. Therefore, although the reported theta effects roughly peak between 300 and $500 \mathrm{msec}$ (i.e., around the time of lexical access), the latency of the effects cannot be meaningfully related to the timing of the underlying neural processes.

It is worth noting that the present item set contained OC words across many semantic categories (recall that the stimulus material consisted of a short story). Therefore, reasoning along the lines of Pulvermüller (1999), one would have expected a widespread theta power increase because in his view the meaning of a word at least partly determines the cortical topography of its representation (see Martin \& Chao, 2001, for similar propositions). Although a widespread theta power increase following OC words is not entirely incompatible with our results (we found a theta increase that was overall larger following OC words, expressed in a main effect of word class), the focal increase at left temporal sites for OC words argues against such a semantically determined cortical topography. This issue could be further clarified by performing experiments that use word lists of, for example, nouns pertaining to different semantic categories (such as auditory, visual, and somatomotor). Such experiments could be useful in determining whether words belonging to different semantic categories elicit theta responses with different scalp topographies (temporal, occipital, or central, respectively).

As a last remark, the scalp topographies of all three frequency components are markedly different from the scalp topographies of the different ERP components reported in the ERP analysis of the same data (Brown et al., 1999). There an early left-dominant anterior negativity, a right parietal $\mathrm{N} 400$ for $\mathrm{OC}$ words, and a widely distributed left-lateralized N400-700 were found. As opposed to the results obtained from most ERP studies, where CC items elicit ERP components (e.g., the N400700) that are not elicited by OC items, the present timefrequency analysis does not show any effects that are unique to CC items, nor even components that are stronger for CC items than for OC items. This holds for all three frequency bands in which we found significant effects. It is therefore unlikely that the reported effects index brain activity that is a result of the syntactic operations induced by CC items. The discrepancy between the results of the ERP analysis and those of the present frequency-domain analysis again underlines the fact that the different measures are a reflection of different aspects of neural activity. Several attempts have been made to characterize these differences in functional terms. Some have taken the stance that ERPs are nothing but a superposition of oscillations in different frequency bands, which enter into transient phase locking (e.g., Makeig et al., 2002; Basar, Schurman, Demiralp, Basar-Eroglu, \& Ademoglu, 2001), but this seems to be a rather extreme viewpoint. Others have argued that synchronous oscillations are particularly instrumental in top-down processing (e.g., Engel, Fries, \& Singer, 2001; Tallon-Baudry \& Bertrand, 1999) or in a largescale integration of bottom-up and top-down processes (Varela et al., 2001). However, empirical underpinning of these ideas is still lacking. Essentially, despite the clear differences in terms of signal properties and corresponding analysis methodologies, the exact functional relationship between ERPs and oscillatory EEG components is only poorly understood at present.

In conclusion, we have found evidence for the emergence of synchronous oscillatory neural activity in the theta frequency range in distinct parts of the brain during language processing. This suggests that theta oscillations play a functional role in binding the different sources of information needed for understanding the linguistic input. The qualitative differences in lowfrequency (theta band) oscillatory neural activity between OC words and CC words support the notion that elements from the two word classes are represented and/or accessed in qualitatively different ways, although 
alternative explanations cannot be excluded at this stage. In addition, together with the few other studies that investigated oscillatory dynamics during language processing, our data are compatible with the notion of a functional role for oscillatory synchrony in binding together different aspects of the linguistic input to obtain a unified and coherent comprehension of the input. Interestingly, and in contrast with the propositions put forward by Pulvermüller (2000), we found evidence for increased synchrony in the lower (i.e., theta) frequencies. Together with previous reports of increased beta (Weiss \& Rappelsberger, 1996) and gamma (Pulvermüller, Lutzenberger, \& Preissl, 1999) coherence during language comprehension, the data suggest a functional role of both fast and slow oscillations in language comprehension.

\section{METHODS}

\section{Participants}

Thirteen students ( 6 men, 7 women) from the University of Nijmegen (aged 21-28 years), were paid for their participation after having given informed consent according to the Declaration of Helsinki. All were native Dutch speakers with normal or corrected-to-normal vision. They were right-handed, as assessed by an abridged Dutch version of the Oldfield Handedness Inventory (Oldfield, 1971). None of the participants had any neurological impairment or neurological trauma, and none used neuroleptics.

\section{Stimulus Material}

Stimulus materials consisted of a simple Dutch fairy-talelike story. The text contained 151 sentences, with an average length of 10.2 words (range, 4-16). Active sentences made up $92 \%$ of the story. The text contained 1540 words and included words from a diversity of syntactic categories. From this set of words, 439 OC words (202 nouns, 86 adjectives, 151 verbs) and 398 CC words (212 articles, 115 prepositions, 71 conjunctions) were selected for analysis. The CC items consisted of 23 different items (average repetition rate per item in the experiment was 17.3 times), the OC items consisted of 355 different items (average repetition rate per item was 1.24 for the experiment).

Sentence-initial and sentence-final words were not included in this set to avoid sentence start-up and wrap-up effects. Words were randomly selected from all other serial positions within a sentence.

The selected OC words ranged in length from 3 to 12 characters and their frequency of occurrence (in a million words) was between 0.0 and 4.3 on a $\log$ frequency scale. The selected CC words ranged in length from 2 to 5 characters and their frequency of occurrence was between 2.42 to 4.44 . Word frequencies, obtained from the digital lexicon CELEX (Baayen, Piepenbrock, \& van Rijn, 1993) were grouped in bins with a width of $0.2 \mathrm{log}$ frequency (see Figure 3). For the statistical analyses, we used word scarcity, defined as $5-\log$ frequency. This transformation ensures that the predictor is positively rather than negatively correlated with the dependent measure.

\section{Procedure}

Participants were seated in a comfortable reclining chair in a sound-attenuating room. The text was presented visually, word by word, on a high-resolution PC screen in a $10-$ by $2.5-\mathrm{cm}$ window cut out of a black nonreflecting shield covering the screen. Each word lasted on screen for $400 \mathrm{msec}$ and was followed by a 400 -msec blankscreen interval. A completed sentence was followed by a blank-screen interval of $2200 \mathrm{msec}$. Sentence-initial words were written with an initial capital letter; all other letters were in lowercase. Sentence-final words appeared together with a period, question mark, or exclamation mark, as appropriate. Participants were instructed not to blink during the sentence presentation, but to blink only in the interval between sentences. The viewing distance was between 70 and $80 \mathrm{~cm}$, and the stimuli subtended a vertical visual angle of approximately $3^{\circ}$.

Participants were instructed to read the text carefully for comprehension, with no additional task. The test story was preceded by a short practice story to familiarize the participants with the experimental procedure. The test story was then presented in four blocks of approximately 5 minutes each. To make sure that the participants were actually reading the text, the experimenter would question the participants about the story

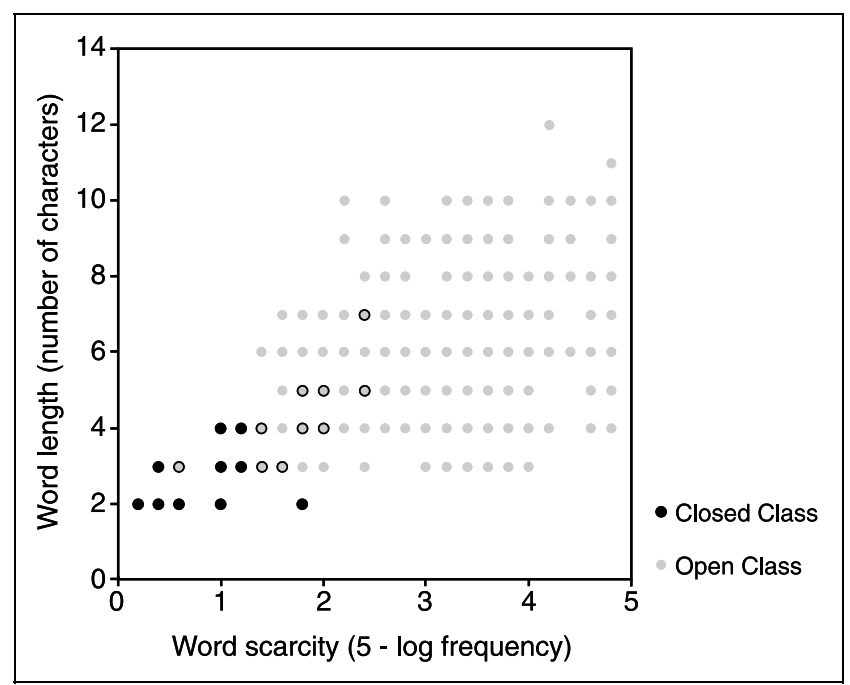

Figure 3. Scatterplot of the lexicostatistical properties of the stimuli, given separately for OC (gray circles) and CC (black circles) words. Black circles with gray fillings indicate the presence of both OC and CC words. 
at various moments during the breaks between the blocks. A session lasted approximately $2 \mathrm{hr}$, including electrode application and removal.

\section{EEG Recordings}

EEG was recorded from 29 tin electrodes attached to an electrode cap (see Figure 4 for the electrode configuration). A reference electrode was placed on the left mastoid. Vertical and horizontal eye movements were monitored bipolarly via sub- and supraorbital electrodes and left and right external canthal montages, respectively. The ground electrode was placed at $\mathrm{Cz}$. Impedances were kept below $4 \mathrm{k} \Omega$. The EEG and EOG recordings were amplified using a band-pass filter of 0.016 to $35 \mathrm{~Hz}$, digitized on-line with a sample frequency of $200 \mathrm{~Hz}$, and stored for off-line analysis.

The continuous EEG was segmented into epochs of $1600 \mathrm{msec}$, lasting from $750 \mathrm{msec}$ before word onset until $850 \mathrm{msec}$ after word onset. These epochs were visually inspected for artifacts (eye blinks, horizontal and vertical eye movements, muscle artifacts, etc.). After removing trials with artifacts, an average of 404 and 366 epochs per participant remained in the OC and CC conditions, respectively.

\section{Time-Frequency Analysis}

To isolate the induced-type oscillations from the (evoked-type) ERP components (cf. Tallon-Baudry \& Bertrand, 1999; Bullock, 1992, for descriptions of this distinction), we computed, for every subject, the average ERP, and subtracted this average from each single trial.

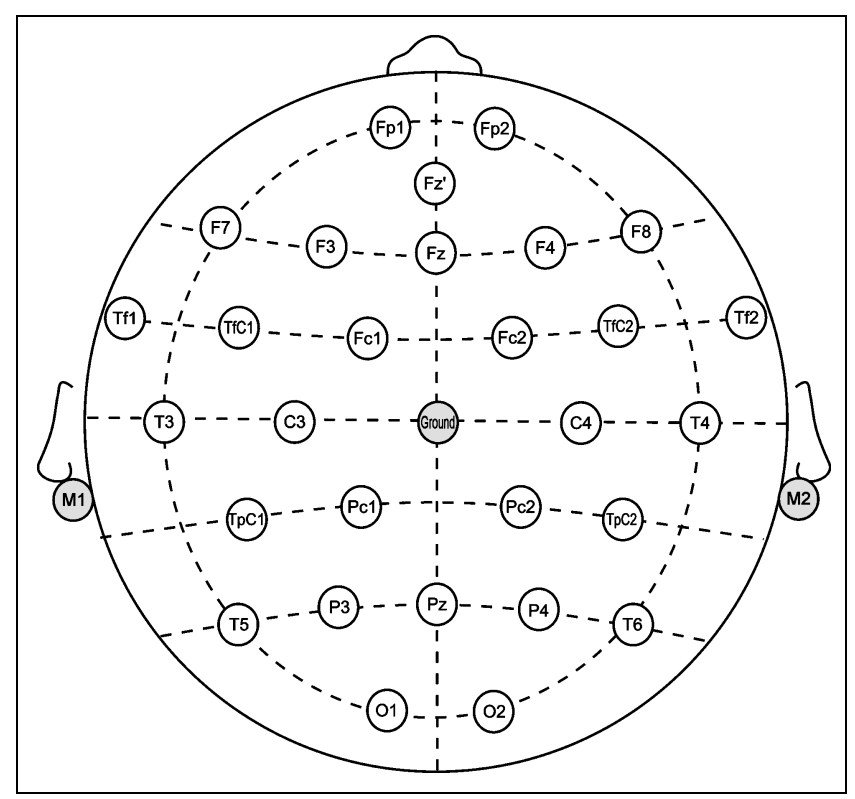

Figure 4. Radial projection of the electrode montage and corresponding electrode labels.
This was done separately for the two word classes (e.g., Klimesch, Russegger, Doppelmayr, \& Pachinger, 1998; Kalcher \& Pfurtscheller, 1995, for a similar approach).

TFRs of the single trial data were then obtained by computing the squared norm of the result of the convolution of complex Morlet wavelets with the EEG data, as described by Tallon-Baudry et al. (1996). We used wavelets with a seven-cycle width, with frequencies ranging from 1 to $30 \mathrm{~Hz}$, in $1-\mathrm{Hz}$ steps. Note that because of the filter settings $(0.016$ to $35 \mathrm{~Hz})$ used in the EEG recordings, we were not able to analyze activity in the gamma frequency range. Power values thus obtained were expressed as a percentage change relative to the power in a baseline interval from -150 to $0 \mathrm{msec}$ before word onset. This was done to normalize for (1) individual differences in EEG power and (2) for differences in power between different frequency bands (e.g., alpha frequencies usually contain more power than, e.g., theta frequencies. This may lead to seemingly larger reactivity (increases or decreases) of the power in alpha frequencies if one takes absolute power instead of percent change as the dependent measure).

\section{Acknowledgments}

This research was supported by grant 400-56-384 of the Netherlands Organisation for Scientific Research (NWO). At the time of manuscript preparation, M.B. was supported by Human Frontier Science Program grant HFSP148/2000. The authors thank Peter Indefrey and Pascal Fries for their comments on an earlier version of this article.

Reprint requests should be sent to Dr. Marcel C.M. Bastiaansen, FC Donders Centre for Cognitive Neuroimaging, Trigon 181, PO Box 9101, 6500 HB Nijmegen, The Netherlands, or via email: marcel.bastiaansen@fcdonders.kun.nl.

\section{REFERENCES}

Baayen, R. H., Piepenbrock, R., \& van Rijn, H. (1993). The CELEX lexical database (CD-ROM). Philadelphia: University of Pennsylvania.

Basar, E., Schurmann, M., Demiralp, T., Basar-Eroglu, C., \& Ademoglu, A. (2001). Event-related oscillations are "real brain responses"-wavelet analysis and new strategies. International Journal of Psychophysiology, 39, 91-127.

Bastiaansen, M. C. M., \& Brunia, C. H. (2001). Anticipatory attention: An event-related desynchronization approach. International Journal of Psychophysiology, 43, 91-107.

Bastiaansen, M. C. M., \& Hagoort, P. (2003). Event-induced theta responses as a window on the dynamics of memory. Cortex, 39, 967-992.

Bastiaansen, M. C. M., Posthuma, D., Groot, P. F., \& de Geus, E. J. (2002). Event-related alpha and theta responses in a visuo-spatial working memory task. Clinical Neurophysiology, 113, 1882-1893.

Bastiaansen, M. C. M., van Berkum, J. J., \& Hagoort, P. (2002a). Event-related theta power increases in the human EEG during online sentence processing. Neuroscience Letters, 323, 13-16.

Bastiaansen, M. C. M., van Berkum, J. J., \& Hagoort, P. (2002b). Syntactic processing modulates the theta rhythm of the human EEG. Neuroimage, 17, 1479-1492. 
Benson, D. F. (1979). Neurologic correlates of anomia. In H. Whitaker \& H. A. Whitaker (Eds.), Studies in neurolinguistics (vol. 4, pp. 293-328). New York: Academic Press.

Binder, J. R., Frost, J. A., Hammeke, T. A., Rao, S. M., \& Cox, R. W. (1996). Function of the left planum temporale in auditory and linguistic processing. Brain, 119, 1239-1247.

Bradley, D. C., \& Garrett, M. F. (1983). Hemisphere differences in the recognition of closed and open class words. Neuropsychologia, 21, 155-159.

Brown, C. M., Hagoort, P., \& ter Keurs, M. (1999). Electrophysiological signatures of visual lexical processing: Open and closed-class words. Journal of Cognitive Neuroscience, 11, 261-281.

Bullock, T. H. (1992). Introduction to induced rhythms: A widespread, heterogeneous class of oscillations. In E. Basar \& T. H. Bullock (Eds.), Induced rbythms in the brain (pp. 1-26). Boston: Birkhauser.

Burgess, A. P., \& Ali, L. (2002). Functional connectivity of gamma EEG activity is modulated at low frequency during conscious recollection. International Journal of Psychophysiology, 46, 91-100.

Burgess, A. P., \& Gruzelier, J. H. (2000). Short duration power changes in the EEG during recognition memory for words and faces. Psychophysiology, 37, 596-606.

Chiarello, C., \& Nuding, S. (1987). Visual field effects for processing content and function words. Neuropsychologia, 25, 539-548.

Cohen, L., Dehaene, S., Naccache, L., Lehericy, S., Dehaene-Lambertz, G., Henaff, M. A., \& Michel, F. (2000). The visual word form area: Spatial and temporal characterization of an initial stage of reading in normal subjects and posterior split-brain patients. Brain, 123, 291-307.

Engel, A. K., Fries, P., \& Singer, W. (2001). Dynamic predictions: Oscillations and synchrony in top-down processing. Nature Reviews Neuroscience, 2, 704-716.

Fell, J., Klaver, P., Lehnertz, K., Grunwald, T., Schaller, C., Elger, C. E., \& Fernandez, G. (2001). Human memory formation is accompanied by rhinal-hippocampal coupling and decoupling. Nature Neuroscience, 4, 1259-1264.

Foxe, J. J., Simpson, G. V., \& Ahlfors, S. P. (1998). Parieto-occipital $\sim 10 \mathrm{~Hz}$ activity reflects anticipatory state of visual attention mechanisms. NeuroReport, 9, 3929-3933.

Friederici, A. D. (1983). Aphasics' perception of words in sentential context: Some real-time processing evidence. Neuropsychologia, 21, 351-358.

Friederici, A. D. (1985). Levels of processing and vocabulary types: Evidence from on-line comprehension in normals and agrammatics. Cognition, 19, 133-166.

Fries, P., Reynolds, J. H., Rorie, A. E., \& Desimone, R. (2001). Modulation of oscillatory neuronal synchronization by selective visual attention. Science, 291, 1560-1563.

Gevins, A., Smith, M. E., McEvoy, L., \& Yu, D. G. (1997). High-resolution EEG mapping of cortical activation related to working memory: Effects of task difficulty, type of processing, and practise. Cerebral Cortex, 7, 374-385.

Gray, C., König, P., Engel, A., \& Singer, W. (1988). Oscillatory responses in cat visual cortex exhibit inter-columnar synchronization which reflects global stimulus properties. Nature, 338, 334-337.

Hagoort, P., Hald, L., Bastiaansen, M., \& Petersson, K. M. (2004). Integration of word meaning and world knowledge in language comprehension. Science, 304, 438-441.

Hagoort, P., Indefrey, P., Brown, C., Herzog, H., Steinmetz, H., \& Seitz, R. J. (1999). The neural circuitry involved in the reading of German words and pseudowords: A PET study. Journal of Cognitive Neuroscience, 11, 383-398.

Indefrey, P., \& Levelt, W. J. M. (1999). The neural correlates of language production. In M. S. Gazzaniga (Ed.), The new cognitive neurosciences (2nd ed., pp. 845-867). Cambridge: MIT Press.

Jensen, O., \& Tesche, C. D. (2002). Frontal theta activity in humans increases with memory load in a working memory task. European Journal of Neuroscience, 15 , 1395-1399.

Kaan, E., \& Swaab, T. Y. (2002). The brain circuitry of syntactic comprehension. Trends in Cognitive Sciences, 6, 350-356.

Kahana, M. J., Seelig, D., \& Madsen, J. R. (2001). Theta returns. Current Opinion in Neurobiology, 11, 739-744.

Kahana, M. J., Sekuler, R., Caplan, J. B., Kirschen, M., \& Madsen, J. R. (1999). Human theta oscillations exhibit task dependence during virtual maze navigation. Nature, 399, 781-784.

Kalcher, J., \& Pfurtscheller, G. (1995). Discrimination between phase-locked and non-phase-locked event-related EEG activity. Electroencephalography and Clinical Neurophysiology, 94, 381-384.

King, J. W., \& Kutas, M. (1995). A brain potential whose latency indexes the length and frequency of words. CRL Newsletter, 10, 1-9.

Kirk, I. J., \& Mackay, J. C. (2003). The role of theta-range oscillations in synchronising and integrating activity in distributed mnemonic networks. Cortex, 39, 993-1008.

Klimesch, W. (1999). EEG alpha and theta oscillations reflect cognitive and memory performance: A review and analysis. Brain Research Reviews, 29, 169-195.

Klimesch, W., Doppelmayr, M., Stadler, W., Pollhuber, D., Sauseng, P., \& Rohm, D. (2001). Episodic retrieval is reflected by a process-specific increase in human electroencephalographic theta activity. Neuroscience Letters, 302, 49-52.

Klimesch, W., Russegger, H., Doppelmayr, M., \& Pachinger, T. (1998). A method for the calculation of induced band power: Implications for the significance of brain oscillations. Electroencephalography and Clinical Neurophysiology, 108, 123-130.

Knosche, T. R., \& Bastiaansen, M. C. (2002). On the time resolution of event-related desynchronization: A simulation study. Clinical Neurophysiology, 113, 754-763.

Lachaux, J. P., Rodriguez, E., Martinerie, J., \& Varela, F. J. (1999). Measuring phase synchrony in brain signals. Human Brain Mapping, 8, 194-208.

Lopes da Silva, F. H. (1991). Neural mechanisms underlying brain waves: From neural membranes to networks. Electroencephalography and Clinical Neurophysiology, 79, 81-93.

Makeig, S., Westerfield, M., Jung, T. P., Enghoff, S., Townsend, J., Courchesne, E., \& Sejnowski, T. J. (2002). Dynamic brain sources of visual evoked responses. Science, 295 , 690-694.

Martin, A., \& Chao, L. L. (2001). Semantic memory and the brain: Structure and processes. Current Opinion in Neurobiology, 11, 194-201.

Miller, R. (1991). Cortico-hippocampal interplay and the representation of contexts in the brain. Berlin: Springer.

Mohr, B., Pulvermüller, F., \& Zaidel, E. (1994). Lexical decision after left, right and bilateral presentation of content words, function words and non-words: Evidence for interhemispheric interaction. Neuropsychologia, 32, 105-124.

Münte, T. F., Wieringa, B. M., Weyerts, H., Szenkuti, A., Matzke, M., \& Johannes, S. (2001). Differences in brain potentials to 
open and closed class words: Class and frequency effects. Neuropsychologia, 39, 91-102.

Neville, H. J., Mills, D. L., \& Lawson, D. S. (1992). Fractioning language: Different neural subsystems with different sensitive periods. Cerebral Cortex, 2, 244-258.

Nobre, A. C., \& McCarthy, G. (1994). Language related ERPs-Scalp distributions and modulation by word type and semantic priming. Journal of Cognitive Neuroscience, 6, 233-255.

Nobre, A. C., \& McCarthy, G. (1995). Language-related field potentials in the anterior-medial temporal lobe: II. Effects of word type and semantic priming. Journal of Neuroscience, 15, 1090-1098.

Nunez, P. L. (1995). Neocortical dynamics and buman EEG rhythms. New York: Oxford University Press.

Oldfield, R. C. (1971). The assessment of the analysis of handedness: The Edinburgh inventory. Neuropsychologia, 32, 97-113.

Osterhout, L. (1997). On the brain response to syntactic anomalies: Manipulations of word position and word class reveal individual differences. Brain and Language, 59, 494-522.

Pfurtscheller, G. (1992). Event-related synchronization (ERS): An electrophysiological correlate of cortical areas at rest. Electroencephalography and Clinical Neurophysiology, 83, 62-69.

Pfurtscheller, G., \& Lopes da Silva, F. H. (1999). Event-related EEG-MEG synchronization and desynchronization: Basic principles. Clinical Neurophysiology, 110, 1842-1857.

Price, C., Indefrey, P., \& Van Turennout, M. (1999). The neurocognitive architectures of language. In C. M. Brown \& P. Hagoort (Eds.), The neurocognition of language (pp. 211-240). Oxford: Oxford University Press.

Pulvermüller, F. (1999). Words in the brain's language. Behavioral and Brain Sciences, 22, 253-336.

Pulvermüller, F. (2000). Distributed assemblies, high frequencies and the significance of EEG/MEG recordings. In R. Miller (Ed.), Time and the brain (pp. 241-249). Amsterdam: Harwood.

Pulvermüller, F., Eulitz, C., Pantev, C., Mohr, B., Feige, B., Lutzenberger, W., Elbert, T., \& Birbaumer, N. (1996). High-frequency cortical responses reflect lexical processing: An MEG study. Electroencephalography and Clinical Neurophysiology, 98, 76-85.

Pulvermüller, F., Lutzenberger, W., \& Preissl, H. (1999). Nouns and verbs in the intact brain: Evidence from event-related potentials and high-frequency cortical responses. Cerebral Cortex, 9, 497-506.

Pulvermüller, F., Preissl, H., Lutzenberger, W., \& Birbaumer, N. (1996). Brain rhythms of language: Nouns versus verbs. European Journal of Neuroscience, 8, 937-941.

Rodriguez, E., George, N., Lachaux, J. P., Martinerie, J., Renault, B., \& Varela, F. J. (1999). Perception's shadow: Long-distance synchronization of human brain activity. Nature, 397, 430-433.

Roskies, A. L. (1999). The binding problem; Special issue. Neuron, 24, 7-125.

Salmelin, R., \& Hari, R. (1994). Spatiotemporal characteristics of rhythmic neuromagnetic activity related to thumb movement. Neuroscience, 60, 537-550.

Scott, S. K., Blank, C. C., Rosen, S., \& Wise, R. J. (2000). Identification of a pathway for intelligible speech in the left temporal lobe. Brain, 123, 2400-2406.

Singer, W. (1993). Synchronization of cortical activity and its putative role in information processing and learning. Annual Review of Physiology, 55, 349-374.

Steriade, M., Gloor, P., Llinás, R. R., Lopes da Silva, F. H., \& Mesulam, M. M. (1990). Basic mechanisms of cerebral rhythmic activities. Electroencephalography and Clinical Neurophysiology, 76, 481-508.

Tallon-Baudry, C., \& Bertrand, O. (1999). Oscillatory gamma activity in humans and its role in object representation. Trends in Cognitive Sciences, 3, 151-162.

Tallon-Baudry, C., Bertrand, O., Delpuech, C., \& Pernier, J. (1996). Stimulus specificity of phase-locked and non-phase-locked $40 \mathrm{~Hz}$ visual responses in human. Journal of Neuroscience, 16, 4240-4249.

ter Keurs, M., Brown, C. M., \& Hagoort, P. (2002). Lexical processing of vocabulary class in patients with Broca's aphasia: An event-related brain potential study on agrammatic comprehension. Neuropsychologia, 40, 1547-1561.

ter Keurs, M., Brown, C. M., Hagoort, P., \& Stegeman, D. F. (1999). Electrophysiological manifestations of open- and closed-class words in patients with Broca's aphasia with agrammatic comprehension. An event-related brain potential study. Brain, 122, 839-854.

Tesche, C. D., \& Karhu, J. (2000). Theta oscillations index human hippocampal activation during a working memory task. Proceedings of the National Academy of Sciences, U.S.A., 97, 919-924.

van Petten, C., \& Kutas, M. (1991). Influences of semantic and syntactic context on open- and closed-class words. Memory and Cognition, 19, 95-112.

Varela, F., Lachaux, J. P., Rodriguez, E., \& Martinerie, J. (2001). The brainweb: Phase synchronization and large-scale integration. Nature Reviews. Neuroscience, 2, 229-239.

Weiss, S., Müller, H. M., \& Rappelsberger, P. (2000). Theta synchronization predicts efficient memory encoding of concrete and abstract nouns. NeuroReport, 11, 2357-2361.

Weiss, S., \& Rappelsberger, P. (1996). EEG coherence within the $13-18 \mathrm{~Hz}$ band as a correlate of a distinct lexical organisation of concrete and abstract nouns in humans. Neuroscience Letters, 209, 17-20. 
Copyright of Journal of Cognitive Neuroscience is the property of MIT Press and its content may not be copied or emailed to multiple sites or posted to a listserv without the copyright holder's express written permission. However, users may print, download, or email articles for individual use. 
Copyright of Journal of Cognitive Neuroscience is the property of MIT Press and its content may not be copied or emailed to multiple sites or posted to a listserv without the copyright holder's express written permission. However, users may print, download, or email articles for individual use. 